\title{
Evaluation of the Rain Effects on Gap Acceptance Behavior at Roundabouts by a Logit Model
}

\author{
Dongmin Lee, ${ }^{1}$ Sooncheon Hwang, ${ }^{2}$ Eunhan $\mathrm{Ka}^{3}$ and Chungwon Lee ${ }^{4}{ }^{4}$ \\ ${ }^{1}$ Associate Professor, Department of Transportation Engineering, University of Seoul, 163 Seoulsiripdae-ro, \\ Dongdaemun-gu, Seoul 02504, Republic of Korea \\ ${ }^{2}$ Graduate Student, Department of Transportation Engineering, The University of Seoul, Korea Seoulsiripdaero 163, \\ Dongdaemun-gu, Seoul 02504, Republic of Korea \\ ${ }^{3}$ Researcher, Institute of Construction and Environmental Engineering, Seoul National University, 1 Gwanak-ro, Gwanak-gu, \\ Seoul 08826, Republic of Korea \\ ${ }^{4}$ Associate Professor, Department of Civil and Environmental Engineering, Seoul National University, Korea 1 Gwanak-ro, \\ Gwanak-gu, Seoul 151-744, Republic of Korea
}

Correspondence should be addressed to Chungwon Lee; chungwon@snu.ac.kr

Received 10 May 2018; Accepted 26 June 2018; Published 11 July 2018

Academic Editor: Raffaele Mauro

Copyright (C) 2018 Dongmin Lee et al. This is an open access article distributed under the Creative Commons Attribution License, which permits unrestricted use, distribution, and reproduction in any medium, provided the original work is properly cited.

\begin{abstract}
A roundabout is generally known as an efficient, safe, and environmentally friendly intersection. Since 2010, the Korea government has taken the lead in constructing roundabouts as part of a special project. During that time, many ideas have been put forward to improve the safety, operation, and design of such roundabouts. In terms of improvements, it is particularly important to understand roundabout gap acceptance behavior. As such, we investigated gap acceptance behaviors at four roundabouts based on field observation during both good weather and rainy conditions. Based on the observed data, roundabout critical gaps were estimated, and a logit model for gap acceptance using various roundabout variables was developed to investigate gap acceptance maneuvering at roundabouts. A total of 2,421 data events for gap acceptance were collected from the field observation. Out of these events, $64.6 \%$ of drivers (1,564 drivers) accepted the given gaps and $35.4 \%$ of drivers (857 drivers) rejected them. The values for critical gaps were estimated using several different estimation methods and ranged from 3.3 to 4.7 seconds. The model was developed using four variables including gap size, type of circulating vehicle, traffic volume at the circulating lane, and weather conditions. The developed model shows that a longer gap results in a 3.669 times higher probability of entering roundabouts when the gap is sufficiently great for acceptance than when the gap is smaller. The effects of other variables, such as circulating vehicle types, circulating traffic volume, and weather conditions, are relatively lower than that of a gap size. Rain conditions influenced gap acceptance maneuvering around a roundabout. Drivers need about a 10 percent longer gap to accept entry into roundabouts during rainy conditions, and gap acceptance probabilities are 10 to 20 percent lower for the same given gap time during rainy conditions compared to good weather conditions.
\end{abstract}

\section{Introduction}

A roundabout is generally defined as a form of a circular intersection in which traffic travels counterclockwise around a central island and in which entering traffic must yield to circulating traffic [1]. Roundabouts are generally considered an efficient intersection system to reduce intersection travel time, a safe intersection design to decrease the number of accidents and damage from accidents, and environmentally friendly. As such, many countries have incorporated roundabouts in their road designs, including Korea. Since 2010, roundabouts have been constructed in Korea as part of a special government-led project. After 87 roundabouts were constructed in 2010, about 20 to 100 roundabouts are constructed every year. There have been improvements in safety and efficiency benefits due to such construction. However, there have also been many issues and ideas put forward to improve those roundabouts in terms of safety, operation, and 
TABLE 1: Mean crash reductions of conversion to roundabouts.

\begin{tabular}{lcc}
\hline Country & & Mean Reductions (\%) \\
\hline Australia & All Crashes & Injury Crashes \\
Frances & $41-61 \%$ & $45-87 \%$ \\
Germany & - & $57-78 \%$ \\
Netherlands & $36 \%$ & - \\
United Kingdom & $47 \%$ & - \\
United States & - & $25-39 \%$ \\
\hline
\end{tabular}

Source: [1].

design. Of particular importance for design considerations is an understanding of roundabout gap acceptance behavior.

Although many studies regarding safety and efficiency effects have investigated roundabouts, there were few empirical studies investigating driver behavior at roundabouts. In roundabouts, traffic flows move based on a yield mechanism of the right-of-way principle that circulating traffic has a right-of-way compared to entering traffic. Entering traffic have to yield to circulating traffic inside roundabouts. Due to this roundabout operation principle, the availability of entering into roundabouts, which is a critical element that influences capacity, is determined by considering the gap size of circulating traffic inside the roundabouts. As such, gap acceptance is very significant in roundabouts. In the US Highway Capacity Manual (HCM), the most important elements are the critical gap in circulating traffic and followup time of entering traffic [2].

Therefore, our study investigated gap acceptance behaviors at roundabouts based on field observation. The purpose of this study is to estimate the critical gap for roundabouts by using various estimation methods. We also develop a logit model for gap acceptance using various influencing factors at roundabouts to investigate gap acceptance maneuvers. The gap acceptance maneuver at roundabouts is generally affected by weather conditions including rain and snow. Thus, we developed a logit model for gap acceptance at roundabouts that included weather variables.

\section{Literature Review}

2.1. Roundabouts. The first roundabout was a circle-shaped intersection built in 1905 at Columbus Circle in New York City [1]. This circle shape intersection was called a traffic circle and was designed to enable high-speed entry with priority and slow circulation that yielded to entering traffic. Before traffic volumes significantly increased, traffic circles worked well. However, as traffic volumes increased, traffic circles started to have safety and efficiency problems. Rectifying these problems led to the development of the modern roundabout in the 1960s. The United Kingdom imposed a new rule that required entering traffic to yield and give way to circulating traffic. This rule prohibited vehicles from entering the intersection as incoming traffic until there were sufficient gaps in the circulating traffic. This approach improved the safety and efficiency of circular intersections. Due to the advantages of roundabouts, many countries adopted modern roundabouts as a common circular intersection and have developed extensive design guides and methods to evaluate operational performance. Many studies have evaluated the effects of roundabout adaptation, typically accident reduction effects. In the United States, conversion to a roundabout results in a reduction of $35 \%$ and $76 \%$ in total and injury crashes, respectively. These accident reduction effects are similar to results from studies in other countries, as shown in Table 1.

Meanwhile, there have been recently many studies to overcome disadvantages of standard roundabouts in particular actual circumstance. And they suggested to implicate some alternative types of roundabouts such as a Turboroundabout, an Assembly roundabout, a traffic signal controlled roundabout, a Dog-bone roundabout, and other new types of roundabouts [3]. Out of these alternative types of roundabouts, a Turbo-roundabout was developed and installed at the end of the 1990s in The Netherlands. The Turbo-roundabout is a type of roundabouts to improve twolane roundabouts in safety and capacity through eliminating the necessity of weaving and conflicts by the divided curbs [4-6].

2.2. Critical Gap and Follow-Up Time in Roundabouts. The capacity of a roundabout is determined by three traffic flows around the roundabouts: entering traffic, circulating traffic, and exiting traffic. The capacity of a roundabout decreases as the circulating traffic flow increases. Maneuvering is similar to the effect of a right-turning stream [2]. In the process of estimating capacity, there are two main measures: critical gap in circulating traffic and follow-up time for entry traffic. The critical gap from circulating traffic is the minimum time gap that an entering driver would accept to merge into the circulating lane of the roundabout. A driver rejects any gaps less than the critical gap and accepts any gap that is greater. In US studies, critical gap and follow-up time were 4.5 to 5.1 seconds and 3.2 to 3.4 seconds, respectively, in single roundabouts [7]. In Korea, a field experiment was also conducted to estimate critical gap and follow-up time in roundabouts and found them, estimated using Wu's method, to be 5.6 seconds and 2.3 seconds, respectively [8]. The critical gap and the follow-up time measured from the experiment 
were relatively greater and smaller than the results in other countries.

2.3. Previous Studies of Gap Acceptance Behavior. Due to the importance of gap acceptance maneuvers in unsignalized intersections and roundabouts, methods have been developed to estimate critical gap, including the Raff method, the Ashworth method, the Troubeck method, and the Wu method. The Raff method is a critical gap estimation method using two cumulative distributions of accepted and rejected gaps [9], and the Ashworth method is based on the hypothesis that average critical gap may be evaluated from the mean of accepted gaps [10]. Meanwhile, the Troubeck method estimates critical gap estimation using a maximum likelihood estimation [11] and is applied with an assumption that the distribution of critical gaps follows a log-normal distribution and calculates the probability that the critical gap would be between the largest rejected and accepted gaps. The $\mathrm{Wu}$ method was developed recently and estimates critical gaps based on an equilibrium of a probability concept [12].

These critical gap estimation methods assume, however, that drivers' behavior remains consistent and all drivers are homogeneous in a deterministic approach. As is well known, a deterministic gap acceptance estimation can estimate only the mean critical gap acceptance from the population, and careful drivers may be overrepresented as a result of information loss [13]. To overcome these limitations, a discrete choice model is used to estimate gap acceptance as probability and only the mean value of gap acceptance based on the probability function for success in accepting a gap. Originally in 1981, Daganzo developed a discrete choice model to estimate gap acceptance based on this probability function. This discrete choice model produces the probability function for success in accepting a gap [14]. This discrete choice model to estimate gap acceptance can be formularized based on the distribution of the mean and variance of the gap acceptance function parameter [15]. Mahmassani and Sheffi used a probit model to investigate gap acceptance behavior. They found that the critical gap decreases as long as drivers are waiting for an acceptable gap [16]. The effects of waiting time on gap acceptance were explained by Polus and his research team [17]. A logit model is one of the currently more popular models to explain gap acceptance behavior [18-25].

Weather conditions generally influence driver behaviors such as for speeding, braking maneuvers, gap acceptance behaviors, and other driving behaviors. A FHWA study showed that inclement weather influences such microscopic traffic behavior [26]. This study investigated changes in driver gap acceptance behavior on intersections during rainy weather conditions. They found that there is a more careful driving behavior for left-turn gap acceptance in the rain, and drivers need greater critical gaps. Zohdy and colleagues found that a larger value for the critical gap for a left turn is needed as the intensity of rain increases [18].

2.4. Studies of Gap Acceptance Maneuver in Roundabouts. Investigation of gap acceptance behavior in roundabouts has been conducted thru two approaches. Most of studies estimated critical gap and follow-up time, which are the two key parameters for gap acceptance. Other studies developed roundabout gap acceptance behavior models and there are many different values for critical and follow-up time for roundabouts, which are summarized in Table 2 . Xu and Tian investigated gap acceptance characteristics at roundabouts in California, US [27], and the critical gaps were estimated in the range of 4.5 to 5.3 seconds at eight single roundabouts. They found that circulating flow rate and speed are two major influencing factors for critical gap and follow-up time.

Polus and his colleagues developed a disaggregate logit model to evaluate the waiting time effect on critical gaps at a roundabout [17] and included only two variables: waiting time and gap size. In their study, critical gaps at roundabouts were calculated in the range of 2 to 5 seconds and the waiting time may affect gaps in those ranges. However, gaps lower than 2 seconds were not likely to be accepted because the risk was considered too high.

2.5. Effects of Roundabout Construction in Korea. In Korea, roundabout construction has been led by federal agencies, typically the Presidential Council on National Competitiveness, the Ministry of Land, Transport and Maritime Affairs, the Ministry of Public Administration and Security, National Police Agency, and some research institutes. Currently, there are more than 500 roundabouts that have been constructed with federal government subsidies and with only local government funding. The roundabouts have been considered successful, and the construction of roundabouts continues to accelerate. Half of the roundabouts were constructed in urban areas and the others in rural areas. In most of these roundabouts, the number of crashes and the damage from the crashes were significantly reduced, and intersection traffic operational performance improved. Most residents were also satisfied with the conversion from signalized and unsignalized intersections to roundabouts. Overall, there has been an observed reduction of $46.1 \%$ in total crashes and $66.7 \%$ in fatality as shown in Table 3.

\section{Methodology}

3.1. Data Collection. Field observations for this study were conducted in Seoul, South Korea, at four typical roundabouts designed using the Korean roundabout design guide [15]. Preliminary observations were conducted at 19 roundabouts, and then four roundabouts were selected for study sites after a final consideration of final design layouts and traffic volume. The field observations were conducted via video recording on both sunny and rainy days. Rainy days selected for data collection were days with more than $5 \mathrm{~mm}$ of rainfall. Videos were recorded at time periods from 7:00 A.M. to 9:00 A.M. for morning peak time, from 12:00 P.M. to 2:00 P.M. for afternoon nonpeak time, and from 6:00 P.M. to 8:00 P.M. during evening peak time. Basic characteristics of the four intersections are summarized in Table 4. Data for gap acceptance behaviors were extracted from the recorded video images using video editing equipment to measure more detailed values of gaps. 
TABLE 2: Critical and follow-up headways values in previous studies.

\begin{tabular}{|c|c|c|c|}
\hline Study name & Country & $\begin{array}{c}\text { Critical Gap } \\
(\mathrm{Sec} .)\end{array}$ & $\begin{array}{c}\text { Follow-up headway } \\
\text { (Sec.) }\end{array}$ \\
\hline Abdullah Ahmad & India & $2.5 \sim 3.0$ & - \\
\hline Abrams et al. & US & 2.20 & - \\
\hline Brilon & Germany & $4.07 \sim 4.45$ & $2.89 \sim 2.99$ \\
\hline Cheng Jie & China & $4.1 \sim 5.4$ & - \\
\hline Dahl \& Lee & Canada & $3.90 \sim 5.30$ & $2.10 \sim 4.20$ \\
\hline Feng Xu & US & $4.5 \sim 5.3$ & - \\
\hline Fortuijn & The Netherlands & $3.16 \sim 3.28$ & 2.10 \\
\hline Gazzarri et al. & Italy & $3.54 \sim 4.10$ & $2.52 \sim 2.76$ \\
\hline Guo Ruijun & China & 2.7 & - \\
\hline HCM (2010) & US & 4.1 & - \\
\hline Iraj Bargego & Iran & $2.52 \sim 4.03$ & - \\
\hline KHCM (2013) & South Korea & 3.21 & - \\
\hline Kim, $\mathrm{T}$ & South Korea & $2.42 \sim 2.7$ & - \\
\hline Liang Ren & Australia & $4.6 \sim 4.8$ & - \\
\hline Mensah S. el al. & US & $2.50 \sim 2.60$ & - \\
\hline NCHRP (3-65) & US & $4.2 \sim 5.9$ & - \\
\hline Nicolosi et al. & Italy & $3.19 \sim 3.99$ & $3.15 \sim 2.11$ \\
\hline Park, S & South Korea & $3.7 \sim 4.1$ & - \\
\hline Qu X et al. & Australia & - & - \\
\hline Rodegerdts et al. & US & $3.90 \sim 5.90$ & $2.60 \sim 4.30$ \\
\hline Shweta Rao & India & $1.36 \sim 2.52$ & - \\
\hline Vasconcelos et al. & Portugal & $3.23 \sim 4.50$ & - \\
\hline Vasconcelos et al. & Portugal & $3.37 \sim 4.28$ & $2.08 \sim 2.20$ \\
\hline $\mathrm{Wu}$ & Germany & 4.12 & 2.88 \\
\hline Xu \& Tian & US & $4.50 \sim 5.30$ & $2.30 \sim 2.80$ \\
\hline Zheng et al. & US & $3.80 \sim 5.50$ & $2.30 \sim 3.80$ \\
\hline
\end{tabular}

Sources: [27-37].

TABLE 3: Accident reduction effects of roundabout in Korea.

\begin{tabular}{lccccc}
\hline \multirow{2}{*}{ Before/After Roundabouts } & & \multicolumn{3}{c}{ Average Annual Crashes } \\
& Total & Fatality & Severe Injury & Injury & Minor Injury \\
\hline Before & 571 & 15 & 258 & 274 & 171 \\
After & 308 & 5 & 118 & $-37.6 \%$ & $-41.7 \%$ \\
\hline & $-46.1 \%$ & $-66.7 \%$ & $-54.3 \%$ & 14 & 24 \\
\hline
\end{tabular}

Source: Korean Roundabout Research Center (http://www.roundabout.or.kr/).

Figure 1 shows the maneuvering of gap acceptance at roundabouts according to the sequence of arriving vehicles at the entry lane and circulating vehicles in the circulatory lane. As shown in Figure 1, the difference in the times when circulating vehicles are passing the gap acceptance decision line can be explained as a gap. This decision line is an imaginary line that drivers at the entry lane use to decide whether to enter or not a circulatory lane while looking at oncoming circulatory vehicle traffic. The gap acceptance maneuver is preceded by the decision to accept or decline the circulating vehicle gap as adequate for entry.
This maneuver is illustrated in Figure 1. When entering vehicle $\# 1$ arrives at the yield line (1) in the middle of the gap of the conflicting vehicles (\#1 and \#2), the entering vehicle using gap (a) is rejected because the gap (a) is insufficient to safely enter the roundabout as shown in Figure 1(a). In comparison, the gap between circulating vehicles \#2 and \#3 is sufficiently large that entering vehicle \#1 accepts the gap and leaves the yield line on the entry lane to enter the roundabout. At this time, entering vehicle \#2 is waiting behind vehicle \#1 before vehicle \#1 accepts the gap, and then it might leave immediately after the first vehicle starts to enter a roundabout using 


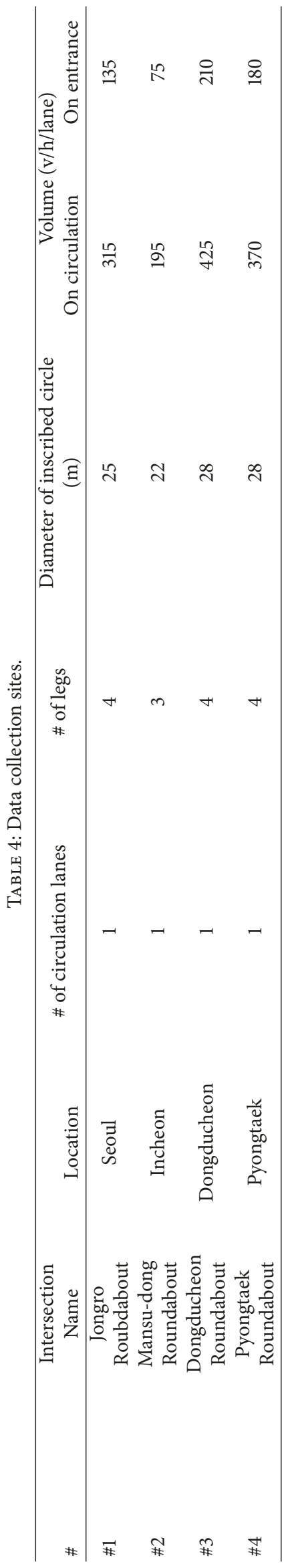




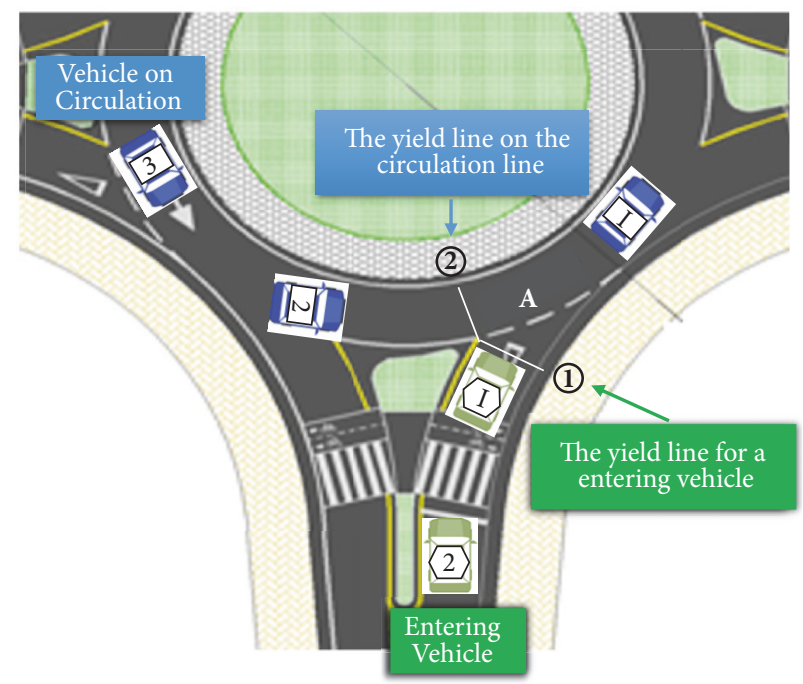

(a) An illustration of sequential gap acceptance maneuvers

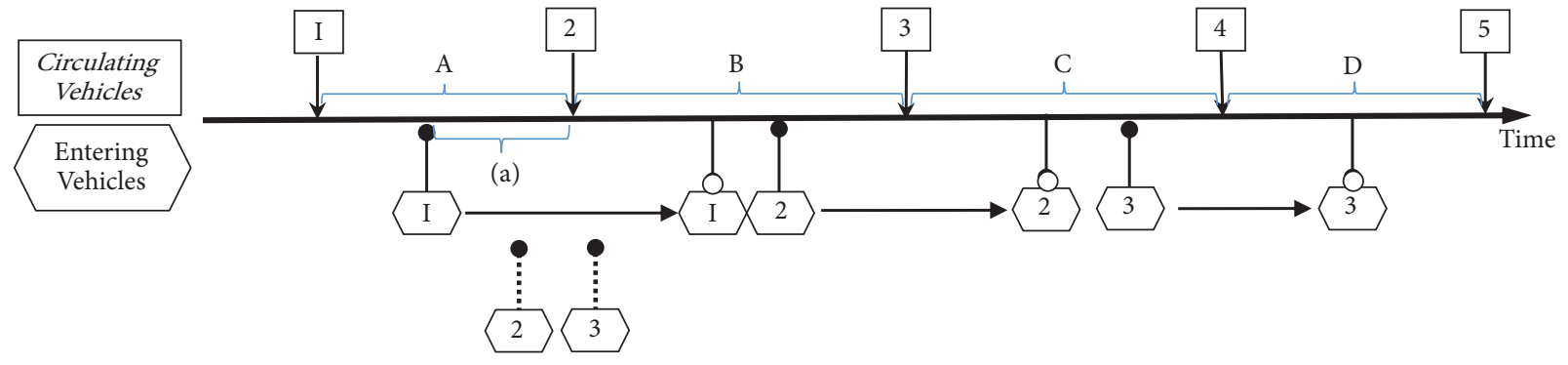

I. Time Interval

$$
\text { A D: Gap }
$$$$
\text { a: Lag }
$$

2. A Vehicle Arriving at Decision Lines (11, (2)

I Circulating-Vehicle

I Entering-Vehicle
3. Times for Vehicle Arriving and Leaving

$\downarrow$ Time when circulating-vehicle passes the line (2) on circulating lane

Time when entering-vehicle arrives at the yield line (1)

Time when entering-vehicle leaves from the yield line (1) for entering

Arrival at the end of queue on approaching lane

(b) The concept of gaps and lags based on time sequence

FIGURE 1: The concept of gap acceptance data extraction from time series maneuvers of roundabouts.

the gap between circulating vehicles \#2 and \#3. Figure 1(b) illustrates the concept of measured gaps and lags based on a time sequence.

3.2. Model Estimation. A gap acceptance maneuver is a binary problem having only two events: success or failure. A driver waiting at the entrance to a roundabout faces a series of binary choices. For each gap at the circulatory lane, the driver has to decide whether to accept it and enter the roundabout or reject it and wait for the next gap. This process continues until a sufficiently large-enough gap occurs, and the driver accepts the gap to enter the roundabout. Therefore, the probability of gap acceptance occurrence under given conditions has to be estimated as dependent variables, which can change due to driving environments instead of the event itself. Since this assists in analysis of the gap acceptance decision-making process and for simplicity and wide applicability, a logit model was selected to model estimation of acceptance probability. This study assumes that all gap acceptance decisions are made independently. For every moment, the driver has a utility from accepting or rejecting a confronting gap. The utility from accepting a gap results from avoiding having to wait 
TABLE 5: Data summary and variable descriptive statistics.

\begin{tabular}{|c|c|c|c|c|}
\hline Category & & Normal & Rainy & Total \\
\hline \multirow{5}{*}{ Gap (sec) } & & 1,681 & 740 & 2,421 \\
\hline & Mean & 5.12 & 5.25 & 5.16 \\
\hline & S.D & 2.81 & 2.97 & 2.86 \\
\hline & Acceptance (\%) & $1,135(67.5 \%)$ & $429(58.0 \%)$ & $1,564(64.6 \%)$ \\
\hline & Reject (\%) & $546(32.5 \%)$ & $311(42.0 \%)$ & $857(35.4 \%)$ \\
\hline \multirow{2}{*}{ Waiting Time (sec) } & Mean & 4.06 & 6.73 & 4.88 \\
\hline & S.D & 5.59 & 8.99 & 6.92 \\
\hline \multirow{3}{*}{ Follow-up time (sec) } & Mean & 17.30 & 15.78 & 16.83 \\
\hline & S.D & 18.62 & 15.90 & 17.84 \\
\hline & Mean & 265.1 & 323.5 & 282.9 \\
\hline \multirow{2}{*}{ \# vehicles observed on Entrance } & S.D & 84.50 & 102.05 & 94.13 \\
\hline & Passenger Car (\%) & $1,498(89.1 \%)$ & $678(91.6 \%)$ & $2,176(89.9 \%)$ \\
\hline \multirow{5}{*}{ \# vehicles observed on Circulation } & Heavy Vehicle (\%) & $183(10.9 \%)$ & $62(8.4 \%)$ & $245(10.1 \%)$ \\
\hline & Mean & 374.5 & 502.6 & 413.6 \\
\hline & S.D & 143.01 & 152.45 & 157.41 \\
\hline & Passenger Car (\%) & $1,466(87.2 \%)$ & $670(90.5 \%)$ & $2,136(88.2 \%)$ \\
\hline & Heavy Vehicle (\%) & $215(12.8 \%)$ & $70(9.5 \%)$ & $285(11.8 \%)$ \\
\hline Weather (\%) & & $1,681(69.4 \%)$ & $740(30.6 \%)$ & - \\
\hline
\end{tabular}

longer to enter the roundabout, whereas the utility from rejecting a gap is the added safety from not accepting a short and dangerous gap. The traffic situation variables that affect these utilities and the decision whether to accept or reject a gap includes traffic volume, waiting time, types of circulating and entering vehicles, and other attributes.

Probability that a driver $\mathrm{n}$ accepts a gap:

$$
\begin{aligned}
P_{n}(\text { accept }) & =P_{n}\left(U_{a n} \geq U_{r n}\right)=\frac{1}{1+e^{-\left(V_{a n}-V_{r n}\right)}} \\
U_{a n} & =V_{a n}+\varepsilon_{a n} \\
U_{r n} & =V_{r n}+\varepsilon_{r n}
\end{aligned}
$$

where $U_{a n}$ and $U_{r n}$ are utility function of accept and reject, respectively; $V_{a n}$ and $V_{r n}$ are the systematic components of the utility of accept and reject, respectively, and are assumed linear in their parameters; $\varepsilon_{a n}$ and $\varepsilon_{r n}$ are the independent and identically Gumbel distributed random components of the utility of accept and reject, respectively.

3.3. Model Validation. Because the logit model in this study produces two outcomes, either "accept" or "reject" for each gap, two different errors type can occur: Type 1 and Type 2. A Type I error is committed if the model predicts that a driver rejects the gap when the driver actually accepts it. Meanwhile, a Type II error is committed if the model predicts that a driver accepts the gap when the driver actually rejects it. The validation for a logit model is generally conducted using the receiver operating characteristics (ROC) curve to test for these two types of errors. ROC curves can show model performance in predicting gap acceptance decisions $[15,38]$. The ROC graph is a $2 \mathrm{D}$ graph having two axes that include a False Positive Rate (FPR) and True Positive Rate (TPR). If the
ROC graph deducted from a model is closer to the point (FDR $=0, \mathrm{TPR}=1$ ), it can be concluded that the developed model is more accurate and is validated. Statistical significance by a numerical value in the ROC graph method can be evaluated thru a C-statistic value. This C-statistic value means the area under the curve (AUC). If the C-statistic value is closer to 1 , it means the ROC curve is close to the point $(\mathrm{FPR}=0 . \mathrm{TPR}=$ 1 ), and means that it is a better model.

\section{Results}

4.1. Data Collection Results. The 2,421 events for gap acceptance were collected from the field observation. Out the total, $64.6 \%$ of drivers (1,564 drivers) accepted the given gaps and $35.4 \%$ of drivers (857 drivers) rejected it as shown in Table 5. The percentage of events for accepted gaps in rainy conditions was relatively smaller than that in good weather conditions at $58.0 \%$ and $67.5 \%$, respectively. Data for 1,681 vehicles were observed under good weather conditions, with 740 vehicles during rainy conditions. About $90 \%$ of cars were classified as a passenger car under good and rain weather conditions. The traffic flow was not congested for the observation, and the average volumes for entering roundabouts and for circulating were 283 vehicles/hour and 414 vehicles/hour, respectively.

To test statistical significance of the difference of gap acceptance between good weather and rain weather conditions, K-S tests were conducted as can be seen in Table 6. This $\mathrm{K}-\mathrm{S}$ test results show that there is statistical difference of gap distributions related weather conditions for both the accepted gap and rejected gap. From these results, it was found that the data analyzed in this study can explain sufficiently the weather's influence on gap acceptance behavior for both gap acceptance and rejection on roundabouts. 
TABLE 6: K-S test results for effects of weather conditions (Rain).

\begin{tabular}{lcc}
\hline Statistics & K-S test for accepted gaps & K-S test for rejected gaps \\
\hline$Z$ & 2.091 & 1.911 \\
$\mathrm{p}$ & $<0.001$ & 0.001 \\
\hline
\end{tabular}

TABLE 7: Critical gaps using various methods.

\begin{tabular}{|c|c|c|c|c|c|}
\hline \multirow{2}{*}{\multicolumn{2}{|c|}{ Location/Time }} & \multicolumn{2}{|c|}{ Raff (Sec.) } & \multicolumn{2}{|c|}{$\mathrm{Wu}(\mathrm{Sec})}$. \\
\hline & & \multirow{2}{*}{$\begin{array}{c}\text { Normal } \\
3.75\end{array}$} & \multirow{2}{*}{$\frac{\text { Rainy }}{4.25}$} & \multirow{2}{*}{$\frac{\text { Normal }}{3.84}$} & \multirow{2}{*}{$\begin{array}{c}\text { Rainy } \\
4.23\end{array}$} \\
\hline & & & & & \\
\hline \multirow{4}{*}{ Location } & $\mathrm{RB} \# 1$ & 4.05 & 4.05 & 4.14 & 4.11 \\
\hline & $\mathrm{RB} \# 2$ & 3.80 & 3.75 & 3.76 & 3.59 \\
\hline & $\mathrm{RB} \# 3$ & 3.70 & 4.05 & 3.80 & 4.12 \\
\hline & RB \#4 & 3.85 & 4.65 & 3.33 & 4.69 \\
\hline \multirow{3}{*}{ Time } & Morning Peak & 3.55 & - & 3.77 & - \\
\hline & PM Non-peak & 4.00 & - & 4.03 & - \\
\hline & Evening Peak & 3.75 & 4.30 & 3.82 & 4.23 \\
\hline
\end{tabular}

TABLE 8: A Logit model development.

\begin{tabular}{|c|c|c|c|c|}
\hline Variables & $\beta$ & S.E, & $\mathrm{P}$ & $\operatorname{Exp}(\beta)$ \\
\hline $\begin{array}{l}\mathrm{X} 1 \\
\text { (Gap, Seconds) }\end{array}$ & 1.300 & 0.059 & $<0.001$ & 3.669 \\
\hline $\begin{array}{l}\text { X2 } \\
\text { (Type of Circulating Vehicles, 0: Passenger Car, 1: } \\
\text { Heavy Vehicle) }\end{array}$ & -0.437 & 0.217 & 0.045 & 0.646 \\
\hline $\begin{array}{l}\text { X3 } \\
\text { (Traffic Volume at the Circulating lane) }\end{array}$ & -0.003 & 0.000 & $<0.001$ & 0.997 \\
\hline $\begin{array}{l}\text { X4 } \\
\text { (Weather Condition, 0: Good weather, 1: Rainy } \\
\text { Condition) }\end{array}$ & -0.815 & 0.170 & $<0.001$ & 0.443 \\
\hline Constant & -3.158 & 0.274 & $<0.001$ & 0.043 \\
\hline Number of Observations & \multicolumn{4}{|c|}{2,181} \\
\hline Log-likelihood Function & \multicolumn{4}{|c|}{-635.530} \\
\hline Restricted Log-likelihood Function & \multicolumn{4}{|c|}{-1417.961} \\
\hline
\end{tabular}

4.2. Critical Gap Estimation. This study estimated the values of critical gaps using two different estimation methods, the Raff method and Wu method, as shown in Table 7. Critical gap estimation was conducted under normal weather and rainy conditions, as well as for specific data collection times, such as morning peak, P.M. nonpeak, and evening peak time. Critical gaps under rainy conditions were higher than during good weather. As such, rain conditions influenced gap acceptance maneuvering in a roundabout, and drivers needed about an 8 to 13 percent longer gap to accept entry into a roundabout under rainy conditions. These critical gap estimation results are similar to the critical gap times from previous studies in many different countries (Table 2).

4.3. Development of a Logit Model for Gap Acceptance at Roundabouts. A logit model with various independent variables investigates the effects of the factors on gap acceptance at roundabouts. The model parameter coefficients were estimated using the maximum likelihood method. Before developing the model, the dataset was separated into two groups, one for model development and the other for model validation through a random selection process. Out of the total data, about 90 percent of data $(2,181$ data) were used for the model development and other data (240 data) were used for the model validation.

In order to develop the gap acceptance model using a logit model formula, eight variables were originally reviewed, and the variables that are significantly correlated with gap acceptance at a 95\% significance level were selected. The model was finally developed using four variables including a gap size, a type of circulating vehicle, traffic volume at the circulating lane, and weather conditions, and the model development results are shown in Table 8 . The results were as expected, where the utility from accepting a gap increases as the gap increases due to a decrease of the risk in entering roundabouts. The developed model shows that a longer gap results in a 3.669 times higher probability of entering roundabouts when the gap is sufficiently great for acceptance than 
TABLE 9: Validation results using the ROC graph method.

\begin{tabular}{lcccc}
\hline C-Statistic & S.D. & P & \multicolumn{2}{c}{$95 \%$ Confidence Interval } \\
& & & Min & Max \\
\hline 0.936 & 0.016 & $<0.001$ & 0.904 & 0.968 \\
\hline
\end{tabular}

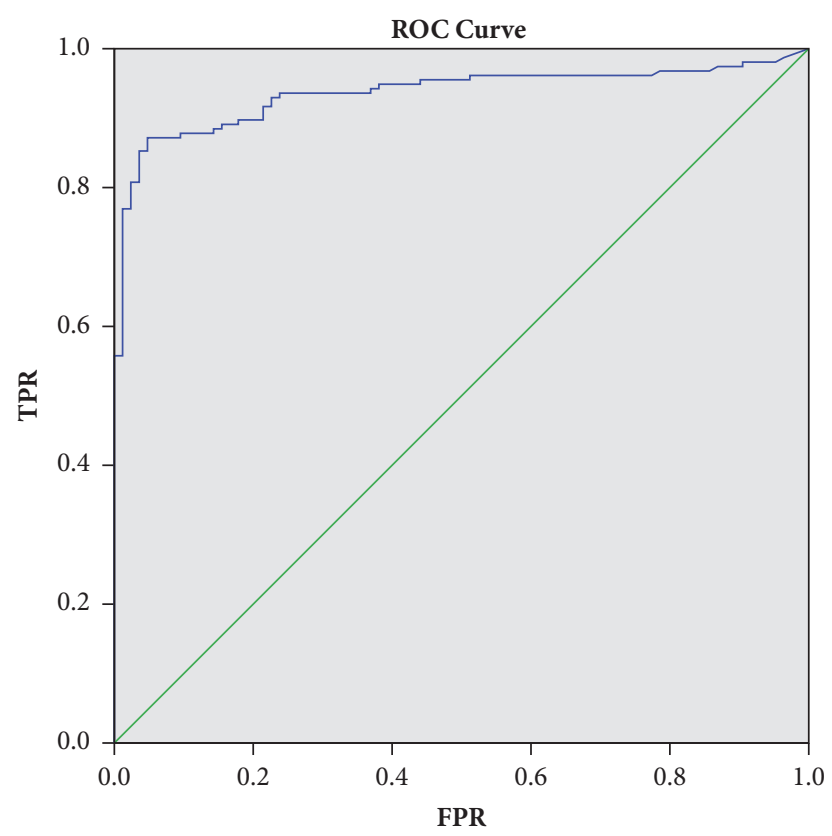

(a)

Figure 2: Deducted ROC graph from the model.

when the gap is smaller. When heavy vehicles are circulated inside roundabouts, drivers have a 0.646 times more difficult time accepting gaps to enter the roundabout compared to when there were only passenger cars inside the roundabouts. When the circulating traffic volume is lower, the probability of gap acceptance increases. Lastly, drivers need a longer gap time to accept when it is raining due to a more conservative driving behavior. In the developed model, there was a 0.443 times lower probability of entering roundabouts when it is raining compared to good weather conditions. Through this model development, the limitation of critical gap estimation that the estimation assumes homogenous driving behaviors for all drivers which is unlikely in real situations can be overcome.

This research used 240 data events out of a total 2,421 data events to validate the developed model. The deducted ROC graph is closer to the point $(\mathrm{FPR}=0$. TPR $=1)$ as shown in Figure 2 and Table 9. The C-statistic value is 0.936 and validates that the results from the developed model are statistically significant and accurate.

Figure 3 shows the estimated probabilities and elasticity of accepting a gap as a function of the gap size for good weather and rain conditions. Figure 3(a) shows the effects of rain conditions on drivers' gap acceptance probabilities. Drivers need about a 10 percent longer gap to accept entry into roundabouts under rainy conditions, and their probabilities of gap acceptance are 10 to 20 percent lower with the same given gap time under rainy conditions than good weather conditions. In the case of three-second gaps, there was a $20 \%$ lower probability in a rainy condition compared to good weather conditions. The elasticity of the logit model represents the responsiveness of an individual's choice probability in regard to a change in the value of some attribute [17]. As Figure 3(b) explains, when the given gaps are less than five seconds, drivers' gap acceptance probabilities in rain conditions did not change more than the change of gap time in good weather conditions because drivers are not willing to enter roundabouts due to riskier conditions during rain. Meanwhile, when the given gaps are greater than five seconds, drivers' acceptance probabilities in rain conditions changed more with a change in gap time.

\section{Conclusions}

A roundabout is generally defined as a form of a circular intersection in which traffic travels counterclockwise around a central island and in which entering traffic must yield to circulating traffic. This roundabout is generally known as an efficient, safe, and environmentally friendly intersection. In Korea, roundabouts have been constructed as a governmentled special project since 2010, and many issues and improvements for roundabouts have been suggested in terms of safety, operation, and design. Of particular importance is understanding gap acceptance behavior in roundabouts.

Gap acceptance behaviors at a roundabout were investigated based on field observations at four roundabouts during both good weather and rainy conditions. Based on the observed data, roundabout critical gaps were estimated, and a logit model for gap acceptance was developed using various variables to investigate gap acceptance maneuvering at roundabouts. Data for 2,421 gap acceptance events were collected from the field observation. Out these events, $64.6 \%$ of drivers (1,564 drivers) accepted the given gaps and 35.4\% of drivers (857 drivers) rejected them. Critical gap values were estimated using the different estimation methods and ranged from 3.3 to 4.7 . These critical gap estimation results are similar to the critical gap times from previous studies in many different countries (Table 3). The model was developed using four variables: gap size, type of circulating vehicle, traffic volume at the circulating lane, and weather conditions. Model validation was conducted using 240 events out of the 2,421 events, which were not used in the model development. For this model validation, the receiver operating characteristics (ROC) curve was tested for two types of errors that occur in binary choice models. This validation process found that the results from the developed model are statistically significant and accurate. This gap acceptance probability model enables overcoming weakness of critical gap estimation that the critical gap estimation assumes homogenous driving behaviors for all drivers unlikely in real situations.

The developed model showed that a longer gap results in a 3.669 times higher probability of entering roundabouts when the gap is sufficiently great for acceptance than when the gap is smaller. The effects of other variables, such as circulating vehicle types, circulating traffic volume, and weather 


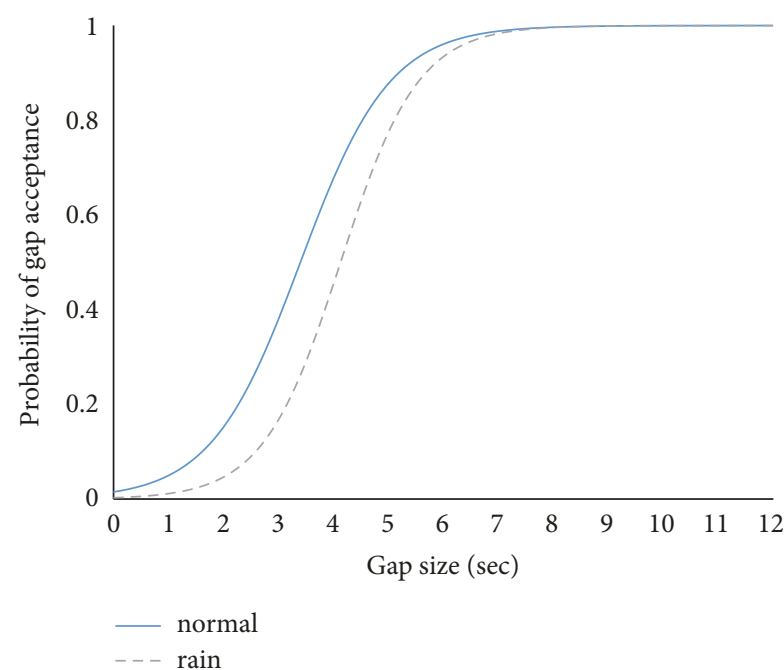

(a) Comparison of gap acceptance probabilities between good weather and rain conditions

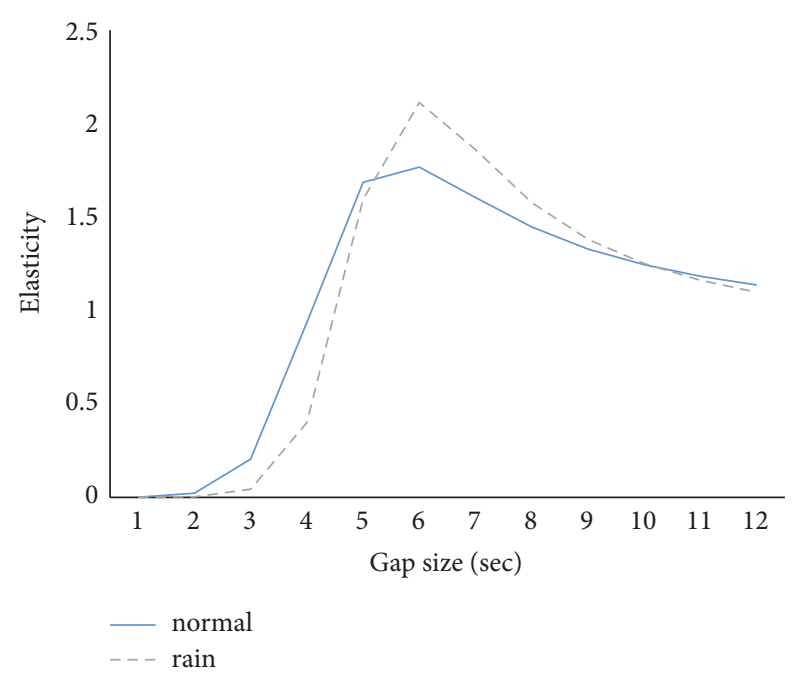

(b) Elastics of gap acceptance between good weather and rain conditions

FIgURE 3: Evaluation of the rain condition effects on gap acceptance behavior.

conditions, are relatively lower than that of a gap size. When heavy vehicles are circulated inside roundabouts, drivers had difficulties reflected in a 0.646 times higher value for accepting gaps for entering roundabouts compared to when there were only passenger cars inside the roundabouts. When there is less circulating traffic, the probability of gap acceptance increases. Lastly, drivers need a longer gap acceptance time when it is raining due to a more conservative driving pattern. The developed model gave a 0.443 times lower probability of entering roundabouts when it is raining compared to good weather conditions. Thus, rain conditions influenced gap acceptance maneuvering into a roundabout. Drivers need about a 10 percent longer gap to accept entering into roundabouts under rainy conditions, and the probabilities of gap acceptance are 10 to 20 percent lower given the same given gap time under rainy conditions than good weather conditions.

This study investigated gap acceptance behaviors at roundabouts by analyzing and estimating critical gaps and the developed a gap acceptance logit model for good weather and rainy conditions. Waiting time was not considered as a statistically significant influence factor based on the analysis results in this study, even though previous studies indicated that waiting time affected gap acceptance behaviors.

There are some limitations to this study, and more field data should be collected as roundabout driving behavior and attitudes mature in Korean. There are still many problems when driving thru roundabouts in Korea due to violations of the right-of-way rule. For more sophisticated weather effect analysis, other types of severe weather conditions such as snow and heavy fog should be considered. Lastly, precipitation may also be an interesting influence factor for gap acceptance behavior analysis.

\section{Data Availability}

The data used to support the findings of this study are available from the corresponding author upon request.

\section{Conflicts of Interest}

The authors declare that they have no conflicts of interest.

\section{Acknowledgments}

This research was partially supported by a Grant [no. 17TLRPB085437-04] from Transportation \& Logistics Research Program funded by Ministry of Land, Infrastructure and Transport of Korean government. This research was partially supported by Institute of Construction and Environmental Engineering at Seoul National University. The authors wish to express their gratitude for the support.

\section{References}

[1] Transportation Research Board, NCHRP Report 672, Roundabouts: An Informational Guide, 2nd edition, 2010.

[2] Transportation Research Board, Highway Capacity Manual, National Research Council, Washington, DC, USA, 2010.

[3] T. Tollazzi, Alternative Types of Roundabouts: An Informational Guide, Springer International Publishing, 2015.

[4] W. Brilon, L. Bondzio, and F. Weiser, "Experiences with turbo roundabouts in Germany," in Proceedings of the National Roundabout Conference, pp. 546-580, Kansas City, MO, USA, 2008.

[5] M. Guerrieri, R. Mauro et al., "Analysis of kinematic parameters and driver behaviour at turbo-roundabouts," Journal of transportation engineering, Part A. Systems, vol. 144, no. 6, 2018.

[6] E. Macioszek, "Analysis of significance of differences between psychotechnical parameters for drivers at the entries to onelane and turbo roundabouts in Poland," in Proceedings of the 
International Conference Transport System Theory and Practice, vol. 505 of Intelligent Transport Systems and Travel Behaviour, Springer International Publishing, Katowice, Poland, 2017.

[7] L. Rodegerdts, M. Blogg et al., "Roundabout in the United States," Tech. Rep. NCHRP Report 572, Transportation Research Board of the National Academics, Washington, DC, USA, 2007.

[8] D. Lee and J. You, "A study on appropriate traffic volume calculation for revitalizing roundabout installation," Journal of Korean Society of Transportation, vol. 31, no. 6, pp. 43-52, 2013.

[9] M. S. Raff and J. W. Hart, "A Volume Warrant for Urban Stop Signs," The Eno Foundation for Highway Traffic Control, 1950.

[10] R. Ashworth, "The capacity of priority-type intersections with a non-uniform distribution of critical acceptance gaps," Transportation Research, vol. 3, no. 2, pp. 273-278, 1969.

[11] R. J. Troutbeck, "Evaluating the performance of a roundabout," Australian Road Research Board, ARRB Group Limited, SR no. 45, 1989.

[12] N. Wu, "Equilibrium of probabilities for estimating distribution function of critical gaps at unsignalized intersections," Transportation Research Record, Journal of the Transportation Research Board, no. 2286, pp. 49-55, 2012.

[13] A. J. Miller, "Nine estimators of gap acceptance parameters," in Proceedings of the International Symposium on the Theory of Traffic Flow and Transportation, G. Newell, Ed., American Elsevier Publishing, Berkeley, Calif, USA, 1972.

[14] C. F. Daganzo, "Estimation of gap acceptance parameters within and across the population from direct roadside observation," Transportation Research Part B: Methodological, vol. 15, no. 1, pp. 1-15, 1981.

[15] X. Chen, Y. Qi, and G. Liu, "Empirical study of gap-acceptance behavior of right-turn-on-red drivers on dual right-turn lanes," Journal of Transportation Engineering, vol. 139, no. 2, pp. 173180, 2013.

[16] H. Mahmassani and Y. Sheffi, "Using gap sequences to estimate gap acceptance functions," Transportation Research Part B: Methodological, vol. 3, no. 15, pp. 143-148, 1981.

[17] A. Polus, Y. Shiftan, and S. Shmueli-Lazar, "Evaluation of the waiting-time effect on critical gaps at roundabouts by a logit model," European Journal of Transport and Infrastructure Research, vol. 5, no. 1, pp. 1-12, 2005.

[18] I. Zohdy, S. Sadek, and H. A. Rakha, "Empirical analysis of effects of wait time and rain intensity on driver left-turn gap acceptance behavior," Transportation Research Board: Transportation Research Record, no. 2173, pp. 1-10, 2010.

[19] T. H. Maze, "A probabilistic model of gap acceptance behavior," Transportation Research Board: Transportation Research Record, no. 795, pp. 8-13, 1981.

[20] S. M. Madanat, M. J. Cassidy, and M.-H. Wang, "Probabilistic delay model at stop controlled intersection," Journal of Transportation Engineering, vol. 120, no. 1, pp. 21-36, 1994.

[21] J. L. Gattis and S. T. Low, "Gap acceptance at atypical stopcontrolled intersections," Journal of Transportation Engineering, vol. 125, no. 3, pp. 201-207, 1999.

[22] P. Liu, X. Wang, J. Lu, and G. Sokolow, "Headway acceptance characteristics of U-turning vehicles at unsignalized intersections," Transportation Research Board: Transportation Research Record, no. 2027, pp. 52-57, 2007.

[23] P. C. Devarasetty, Y. Zhang, and K. Fitzpatrick, "Differentiating between left-turn gap and lag acceptance at unsignalized intersections as a function of the site characteristics," Journal of
Transportation Engineering, ASCE, vol. 138, no. 5, pp. 580-588, 2012.

[24] S. Teply, M. I. Abou-Henidy, and J. D. Hunt, "Gap acceptance behavior-aggregate and logit perspectives: part 1," Traffic Engineering and Control, vol. 38, no. 9, pp. 474-482, 1997.

[25] S. Teply, M. I. Abou-Henidy, and J. D. Hunt, "Gap acceptance behavior-aggregate and logit perspectives: part 2," Traffic Engineering and Control, vol. 38, no. 10, pp. 540-544, 1997.

[26] H. Rakha, K. Daniel, C. Gustave et al., Microscopic Analysis of Traffic Flow in Inclement Weather, FHWA-JPO-09-066, U.S. Department of Transportation, Research and Innovative Technology Administration, 2009.

[27] F. Xu and Z. Z. Tian, "Driver behavior and gap-acceptance characteristics at roundabouts in California," Transportation Research Record: Journal of the Transportation Research Board, vol. 2071, no. 1, pp. 117-124, 2008.

[28] T. Kim, M. Park, and B. Park, "A critical gap model for roundabouts in Korea," Journal of Korean Society of Transportation, vol. 30, no. 2, pp. 93-100, 2012.

[29] S. Park, D. Kim, and J. Jeong, "Evaluation of multi-legged roundabout using surveyed critical gap acceptance," The Journal of the Korea Contents Association, vol. 13, no. 9, pp. 400-409, 2013.

[30] A. Ahmad, R. Rastogi, and S. Chandra, "Estimation of critical gap on a roundabout by minimizing the sum of absolute difference in accepted gap data," Canadian Journal of Civil Engineering, vol. 42, no. 12, pp. 1011-1018, 2015.

[31] J. Cheng, X. Yang, W. Deng, and X. Huang, "Driver's critical gap calibration at urban roundabouts: a case study in China," Tsinghua Science and Technology, vol. 13, no. 2, pp. 237-242, 2008.

[32] R. Guo and B. Lin, "Traffic operation performances at roundabout weaving sections," Journal of Transportation Systems Engineering and Information Technology, vol. 10, no. 3, pp. 2934, 2010.

[33] I. Bargegol, S. H. Hosseini, and M. J. Samet, "Determining the capacity model of urban roundabouts, considering the drivers' behaviour in accepting and rejecting of gaps," in Proceedings of the IOP Conference Series: Materials Science and Engineering, vol. 254, June 2017.

[34] L. Ren, X. Qu, H. Guan et al., "Evaluation of roundabout capacity models: an empirical case study," American Society of Civil Engineers: Journal of Transportation Engineering, vol. 142, no. 12, 2016.

[35] S. Rao, Y. Krishna, P. Atmakuri et al., "Calibration of performance of roundabouts based on gap acceptance parameters using simulation for indian scenario," in Proceedings of the 12th International Conference on Transportation Planning and Implementation Methodologies for Developing Countries (TPMDC '16), pp. 19-21, IIT Bombay, Mumbai, India, 2016.

[36] X. Shi and J. Zhu, "Modification of a gap acceptance theory model of roundabouts' capacities," in Proceedings of the 16th COTA International Conference of Transportation Professionals, pp. 1655-1662, Shanghai, China, July 2016.

[37] O. Giuffrè, A. Granà, and M. L. Tumminello, "Gap-accepteance parameters for roundabouts: a systematic review," European Transport Research Review, vol. 8, no. 2, pp. 1-20, 2016.

[38] T. Fawcett, "An introduction to ROC analysis," Pattern Recognition Letters, vol. 27, no. 8, pp. 861-874, 2006. 


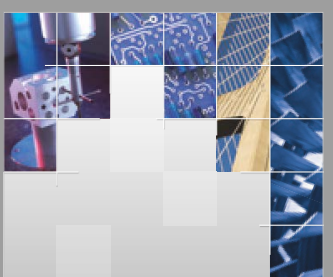

\section{Enfincering}
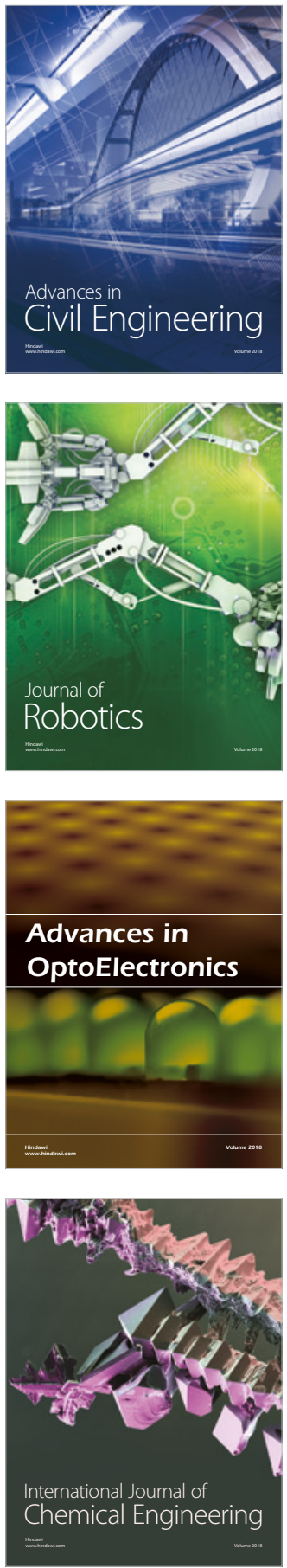

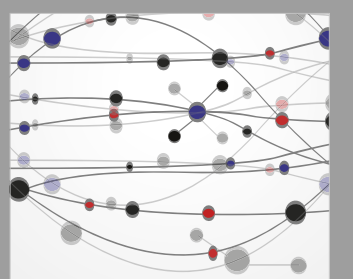

\section{Rotating \\ Machinery}

The Scientific World Journal

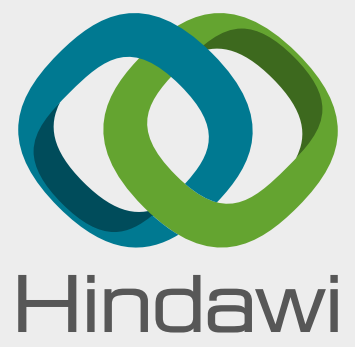

Submit your manuscripts at

www.hindawi.com
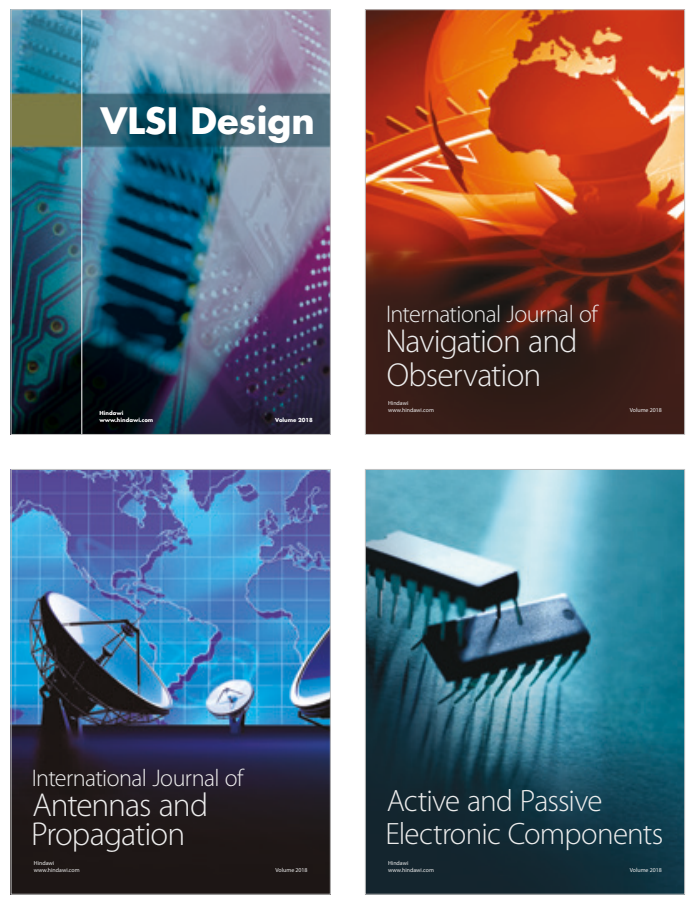
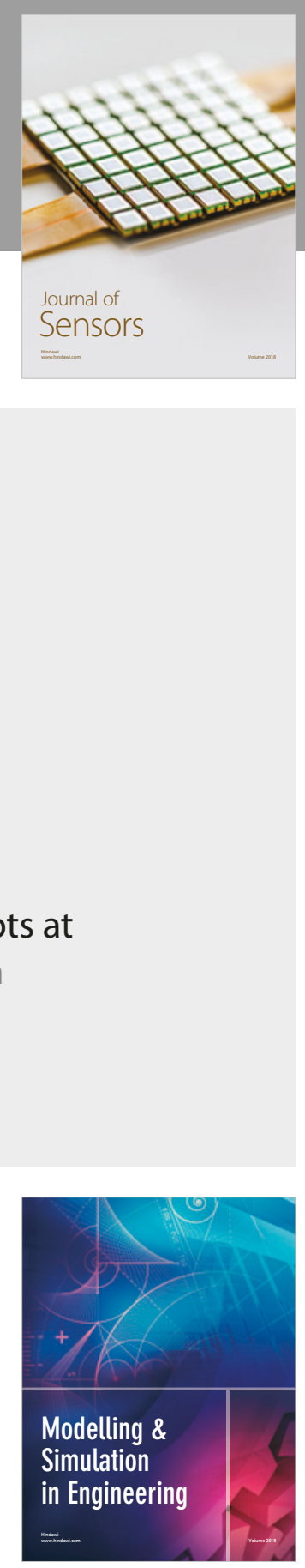

\section{Advances \\ Multimedia}
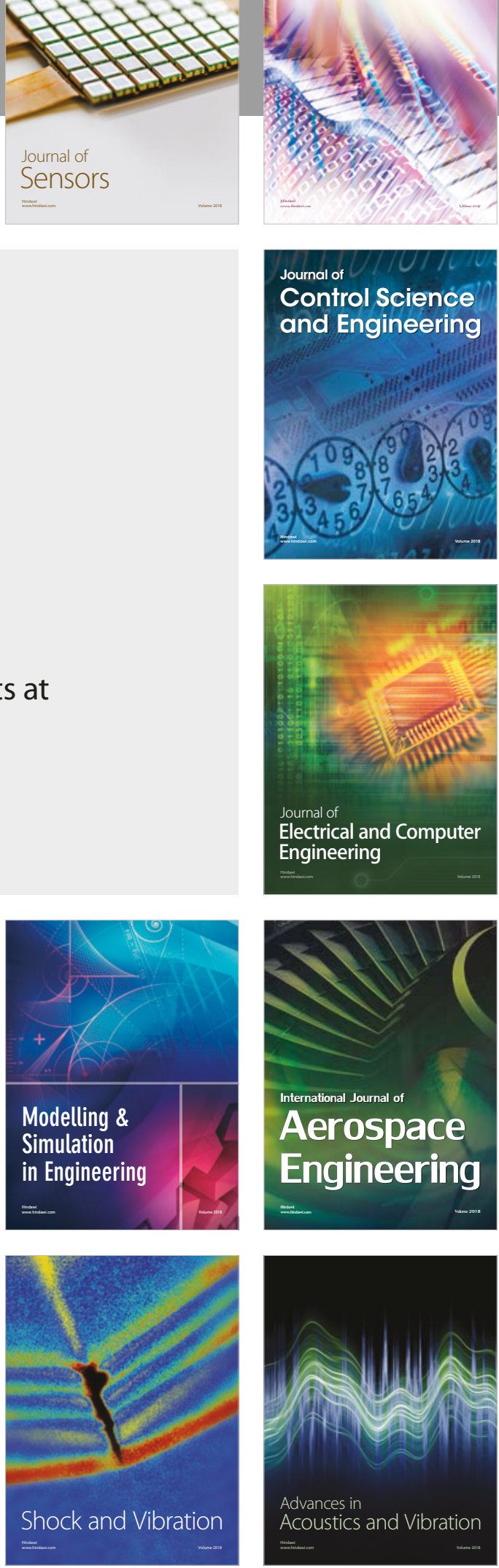